\title{
A new discrete economic model involving generalized fractal derivative
}

\author{
Zhenhua Hu and Xiaokang Tu*
}

"Correspondence:

xiaokangtu@yeah.net

Business School, Central South

University, Changsha, 410083, China

\begin{abstract}
The current article is mainly concerned with applying generalized fractal derivatives in a macroeconomic model. We propose a discrete model involving four macroeconomic variables, the gross domestic production, exchange rate, money supply and exports/imports by using the generalized fractal derivative. The fractal derivative can describe the power-law phenomenon and memory property of economic variables more accurately. Based on the concrete macroeconomic data of Canada, the coefficients of this nonlinear system are estimated by the method of least squares. The statistical test results show that the four variables we have selected have an apparent causal connection, and the sum of squared residuals of the fitting equations is also acceptable. In simulation, the actual data of Canada from 1990 to 2008 are considered, and the effectiveness of our model is verified. The empirical study shows that in the coming few years, the money supply will grow quickly and hence it may lead to proper inflation.
\end{abstract}

MSC: 26A33; 34A08; 00A71; 93E24

Keywords: fractional calculus; fractal derivative; macroeconomic model

\section{Introduction}

The fractal and fractional derivatives can be regarded as the generalization of usual derivatives [1-3]. They are powerful tools in modeling anomalous physical processes, macroand micro-scale phenomena, long-term relation description and many discontinuous problems since they involve extra parameters, the order of derivatives. Selecting different fractional and fractal derivatives and their associated order values leads to various fractional and fractal derivative models. In recent forty years, fractional calculus has gained considerable attention in both applied mathematics and engineering fields such as viscoelastic mechanics, quantum physics, ecology, power-law phenomenon in fluids, electromagnetic field, economics modeling and financial systems. For a comprehensive understanding of this subject and its applications in economic and financial models, we refer readers to [4-16]. The advantage of applying fractional derivative in modeling economic and financial problems is that the non-local property of fractional derivative can depict the memory characteristics in many real economic and financial data $[8,9]$.

Besides the memory characteristics, some of the actual economic and financial data exhibit a power-law property [17-21]. Power law is the form taken by a remarkable number of regularities in economics, and it renders a relation of the expression $Y=k X^{p}$, where $Y$ and $X$ are variables considered, $p$ is called the power-law exponent, and $k$ is a constant 
coefficient. As we observed, many economic laws take the form of power laws, such as macroeconomic scaling laws, the distribution of income, GDP, interest, trading volume, etc. In empirical studies, fractal derivative is an important modeling tool for anomalous diffusion with the power-law property. Fractal derivatives arise from the fractal time-space metric and thus can naturally depict the intrinsic fractal structure of turbulence. In particular, its anomalous diffusion phenomena. To describe the anomalous inner structure of economic and financial models, many dynamical systems are proposed to model the relations of macroeconomic variables. In [22], the study of economic system by using van der Pol equation is discussed. The variation of initial conditions and control parameters of the van der Pol model enables us to understand the periodic, quasiperiodic, and chaotic motion of economic variable considered. In [23, 24], the bifurcation topological structure and the global complicated character of the kind of a nonlinear financial system are studied. A simplified macroeconomic model discussing the investment, interest rate, and price index is proposed, and various evolution results of these economic variables depending on time and parameters are illustrated. There are also many economic models defined by fractional derivatives. In [25], a fractional order financial model based on the fractional Chen system is proposed. It involves the macroeconomic variables, such as investment, interest, and price index, and exhibits the interesting dynamics behavior of them. In [26], the chaos dynamics and chaos control of an economical system have been studied using the sliding mode method. In [27], a delayed fractional-order financial system is proposed and the complex dynamical behaviors of this system are discussed by numerical simulations. In [28], a macroeconomic system model with Caputo fractional derivative (the definition is given in Section 2) is proposed firstly as

$$
\left\{\begin{array}{l}
{ }^{c} D_{0+}^{\alpha_{1}} x(t)=a_{11} x(t)+a_{12} y(t)+a_{13} z(t)+c_{1}, \\
{ }^{c} D_{0+}^{\alpha_{2}} y(t)=a_{21} x(t)+a_{22} y(t)+a_{23} z(t)+c_{2}, \\
{ }^{c} D_{0+}^{\alpha_{3}} z(t)=a_{31} x(t)+a_{32} y(t)+a_{33} z(t)+c_{3},
\end{array}\right.
$$

where $a_{i j}, c_{i}, i, j=1,2,3$, are the coefficients, $\alpha_{1}, \alpha_{2}, \alpha_{3}$ are the order of fractional derivative. The lower terminal in the fractional derivative can be replaced by some other nonzero constant. However, we consider the economic model in this paper, and the $t$ generally denotes the time, hence we consider $t \in[0,+\infty)$. Variables $x, y$, and $z$ indicate the gross domestic product (GDP), inflation, and unemployment, respectively.

We notice that most of the above mentioned works concentrate on the continuous economic and financial models. There are very few works discussing the modeling of actual macroeconomic data using discrete dynamical system. In mathematics, a discrete dynamical system usually describes a recurrence relation, which is also an equation that recursively defines a sequence or multidimensional array of values once one or more initial terms are given. In difference equation, each further term of the sequence or array is defined as a function of the preceding terms. Discrete dynamical models are widely applied in many mathematical and engineering realms [29-31]. In real-world cases, many macroeconomic variables are observed sequently, i.e., daily, monthly or annually, which motivates us to construct a new model by using difference equation involving a generalized fractal derivative. The coupled relation of macroeconomic variables is expressed via a group of difference equations and the power-law property is characterized by the generalized fractal derivative containing two parameters. 
The main contribution of the current paper consists of three aspects. Firstly, the fractal derivative is introduced and a generalized form of fractal derivative is applied to a constructed discrete macroeconomic model to describe the actual data of the given nation. Different from the fractional economic models in literature, the fractal derivative model proposed in this paper can depict the power-law property in macroeconomic series more accurately. Secondly, we consider the actual data of Canada and the effectiveness of our model is verified in simulation. Moreover, the method can be also applied to study the relation of other macroeconomic variables from other countries without many major modifications. Finally, instead of linear terms in model (1), our new discrete model involves nonlinear relation of macroeconomic variables, which is more accurate in describing the actual data. The rest of this paper is organized as follows. In Section 2, the definitions of fractal derivative and its generalized form are given. In Section 3, the discrete macroeconomic model using generalized fractal derivative is discussed, and the coefficients are estimated via the actual macroeconomic data of the given country. In Section 4, we simulate the dynamics of the new model and some useful observations are given. The effective predictions of macroeconomic variables can be obtained by computing the model corresponding to the future time. Finally, the conclusions are drawn in Section 5, and some additional remarks are listed in Section 6.

\section{Fractal derivative and its generalized form}

In $[32,33]$, a class of fractal derivatives is defined as

$$
\frac{* \partial u}{\partial t^{\alpha}}=\lim _{t_{1} \rightarrow t} \frac{u\left(t_{1}\right)-u(t)}{t_{1}^{\alpha}-t^{\alpha}},
$$

where $\alpha>0$, and function $u(t)$ is continuous over the domain. The notation ' $*$ ' indicates that the fractal derivative is different from the common integer-order partial derivative. In what follows, we consider a more generalized form of the above definition:

$$
\frac{* \partial u^{\beta}}{\partial t^{\alpha}}=\lim _{t_{1} \rightarrow t} \frac{u^{\beta}\left(t_{1}\right)-u^{\beta}(t)}{t_{1}^{\alpha}-t^{\alpha}},
$$

where the parameters $\alpha$ and $\beta$ are real numbers. Notice that if $\alpha=\beta=1$, (3) reduces to the first-order derivative of function $u(t)$. In our study, parameters $\alpha$ and $\beta$ can be negative.

The general relationship between fractional calculus and fractals is explored in [34]. It is verified that the fractal dimension of function is shown to be a linear function of the order of fractional integro-differentiation. This motivates us to employ fractal derivative to model the previous fractional relationship discovered between macroeconomic variables $[8,9]$. Theoretically, fractal modeling has been applied in many random processes, e.g., see [35]. However, to the best of authors' knowledge, there is no particular research on the application of fractal derivative modeling in macroeconomic models. It will be important to study economic and financial models further by using fractal derivatives.

There exist different definitions of fractional derivatives such as Riemann-Liouville derivative, Caputo derivative and Riesz potential (see [1], Chapter 2). The RiemannLiouville and Riesz derivatives are frequently used in theoretical analysis, while the Caputo derivative is commonly applied in modeling many real-world problems. The RiemannLiouville, Caputo, and Riesz derivatives of a given continuously differentiable function 
$u(t)$ of order $\alpha$ are, respectively, defined as

$$
\begin{aligned}
\left({ }^{R L} D_{t}^{\alpha} u\right)(t)= & \frac{1}{\Gamma(m-\alpha)} \cdot \frac{d^{m}}{d t^{m}}\left\{\int_{0}^{t}(t-\tau)^{m-\alpha-1} u(\tau) d \tau\right\}, \\
\left({ }^{c} D_{t}^{\alpha} u\right)(t)= & \frac{1}{\Gamma(m-\alpha)} \int_{0}^{t}(t-\tau)^{m-\alpha-1} u^{(m)}(\tau) d \tau, \\
\left({ }^{R} D_{t}^{\alpha} u\right)(t)= & \frac{-1}{2 \cos \left(\frac{\pi \alpha}{2}\right)}\left[\frac{1}{\Gamma(m-\alpha)} \cdot \frac{d^{m}}{d t^{m}}\left\{\int_{0}^{t}(t-\tau)^{m-\alpha-1} u(\tau) d \tau\right\}\right. \\
& \left.+\frac{(-1)^{m}}{\Gamma(m-\alpha)} \cdot \frac{d^{m}}{d t^{m}}\left\{\int_{t}^{1}(\tau-t)^{m-\alpha-1} u(\tau) d \tau\right\}\right],
\end{aligned}
$$

where $m-1<\alpha<m, m \in \mathbb{N}^{+}, 0 \leq t \leq 1$, and the upper and lower terminals 1 and 0 can be replaced by other fixed real numbers. $\Gamma$ denotes the gamma function.

It is easy to notice that the corresponding difference approximations of the above fractional derivatives involve infinitely many terms as step size approaches zero, which is inconvenient in approximate computation. The fractal derivative defined by (3) contains only one term in its difference form, and hence it is more convenient in approximation.

\section{New discrete macroeconomic model}

To derive the discrete model, we consider the equispaced time notes as $t=t_{0}, t_{1}, \ldots, t_{n}$, $t_{n+1}, \ldots$ If we remove the limit operation in (3), we get the difference form of generalized fractal derivative as

$$
\left.\frac{* \partial u^{\beta}}{\partial t^{\alpha}}\right|_{t=t_{n}} \approx \frac{\beta u^{\beta-1}\left(t_{n}\right)\left[u\left(t_{n+1}\right)-u\left(t_{n}\right)\right]}{\alpha t_{n}^{\alpha}\left(t_{n+1}-t_{n}\right)}=\frac{\beta u_{n}^{\beta-1}\left(u_{n+1}-u_{n}\right)}{\alpha h(n h)^{\alpha}}
$$

where $n=0,1,2, \ldots, h=t_{n+1}-t_{n}$ is the step size. Substituting (7) into model (1) and extending it to a four-variable form, we obtain

$$
\left\{\begin{array}{l}
\frac{\beta_{1} x_{n}^{\beta_{1}-1}\left(x_{n+1}-x_{n}\right)}{\alpha_{1} h_{1}\left(n h_{1}\right)^{\alpha}}=a_{11} x_{n}+a_{12} y_{n}+a_{13} z_{n}+a_{14} u_{n}+c_{1}, \\
\frac{\beta_{2} y_{n}^{\beta_{2}-1}\left(y_{n+1}-y_{n}\right)}{\alpha_{2} h_{2}\left(n h_{2}\right)^{\alpha}}=a_{21} x_{n}+a_{22} y_{n}+a_{23} z_{n}+a_{24} u_{n}+c_{2}, \\
\frac{\beta_{3} z_{3}^{\beta_{3}-1}\left(z_{n+1}-z_{n}\right)}{\alpha_{3} h_{3}\left(n h_{1}\right)^{\alpha}}=a_{31} x_{n}+a_{32} y_{n}+a_{33} z_{n}+a_{34} u_{n}+c_{3}, \\
\frac{\beta_{4} u_{n}^{\beta_{4}-1}\left(u_{n+1}-u_{n}\right)}{\alpha_{4} h_{4}\left(n h_{4}\right)^{\alpha_{4}}}=a_{41} x_{n}+a_{42} y_{n}+a_{43} z_{n}+a_{44} u_{n}+c_{4} .
\end{array}\right.
$$

The remaining task is to evaluate the coefficients in model (8). To simplify the expressions, let us denote the parameter sets as

$$
\begin{aligned}
& P_{x}=\left\langle\alpha_{1}, h_{1}, a_{11}, a_{12}, a_{13}, a_{14}, c_{1}, \beta_{1}\right\rangle, \\
& P_{y}=\left\langle\alpha_{2}, h_{2}, a_{21}, a_{22}, a_{23}, a_{24}, c_{2}, \beta_{2}\right\rangle, \\
& P_{z}=\left\langle\alpha_{3}, h_{3}, a_{31}, a_{32}, a_{33}, a_{34}, c_{3}, \beta_{3}\right\rangle, \\
& P_{u}=\left\langle\alpha_{4}, h_{4}, a_{41}, a_{42}, a_{43}, a_{44}, c_{4}, \beta_{4}\right\rangle,
\end{aligned}
$$


then the iteration form of the discrete model (8) is given as

$$
\left\{\begin{array}{l}
x_{n+1}=x_{n}+f_{1}\left(P_{x}, x_{n}, y_{n}, z, u_{n}\right), \\
y_{n+1}=y_{n}+f_{2}\left(P_{y}, x_{n}, y_{n}, z, u_{n}\right), \\
z_{n+1}=z_{n}+f_{3}\left(P_{z}, x_{n}, y_{n}, z, u_{n}\right), \\
u_{n+1}=u_{n}+f_{4}\left(P_{u}, x_{n}, y_{n}, z, u_{n}\right),
\end{array}\right.
$$

where

$$
\begin{aligned}
& f_{1}\left(P_{x}, x_{n}, y_{n}, z, u_{n}\right)=\frac{\alpha_{1} n^{\alpha_{1}} h_{1}^{\alpha_{1}+1}}{\beta_{1} x_{n}^{\beta_{1}-1}}\left(a_{11} x_{n}+a_{12} y_{n}+a_{13} z_{n}+a_{14} u_{n}+c_{1}\right), \\
& f_{2}\left(P_{y}, x_{n}, y_{n}, z, u_{n}\right)=\frac{\alpha_{2} n^{\alpha_{2}} h_{2}^{\alpha_{2}+1}}{\beta_{2} y_{n}^{\beta_{2}-1}}\left(a_{21} x_{n}+a_{22} y_{n}+a_{23} z_{n}+a_{24} u_{n}+c_{2}\right), \\
& f_{3}\left(P_{z}, x_{n}, y_{n}, z, u_{n}\right)=\frac{\alpha_{3} n^{\alpha_{3}} h_{3}^{\alpha_{3}+1}}{\beta_{3} z_{n}^{\beta_{3}-1}}\left(a_{31} x_{n}+a_{32} y_{n}+a_{33} z_{n}+a_{34} u_{n}+c_{3}\right), \\
& f_{4}\left(P_{u}, x_{n}, y_{n}, z, u_{n}\right)=\frac{\alpha_{4} n^{\alpha_{4}} h_{4}^{\alpha_{4}+1}}{\beta_{4} u_{n}^{\beta_{4}-1}}\left(a_{41} x_{n}+a_{42} y_{n}+a_{43} z_{n}+a_{44} u_{n}+c_{4}\right) .
\end{aligned}
$$

Let $\tilde{x}_{n}, \tilde{y}_{n}, \tilde{z}_{n}$, and $\tilde{u}_{n}$ be the actual data of GDP, exchange rate, money supply and exports/imports, respectively, where $n=1,2, \ldots, N$, and $N$ is the length of the macroeconomic variable. To apply the method of least squares, it suffices to solve the following minimizing problem:

$$
\operatorname{Min} \sum_{n=1}^{N}\left\{\left|\tilde{x}_{n}-x_{n}\right|^{2}+\left|\tilde{y}_{n}-y_{n}\right|^{2}+\left|\tilde{z}_{n}-z_{n}\right|^{2}+\left|\tilde{u}_{n}-u_{n}\right|^{2}\right\}
$$

subject to $x_{n+1}=x_{n}+f_{1}\left(P_{x}, x_{n}, y_{n}, z, u_{n}\right)$,

$$
\begin{aligned}
& y_{n+1}=y_{n}+f_{2}\left(P_{y}, x_{n}, y_{n}, z, u_{n}\right), \\
& z_{n+1}=z_{n}+f_{3}\left(P_{z}, x_{n}, y_{n}, z, u_{n}\right), \\
& u_{n+1}=u_{n}+f_{4}\left(P_{u}, x_{n}, y_{n}, z, u_{n}\right),
\end{aligned}
$$

where the corresponding data in the first year is regarded as the initial conditions.

\section{Empirical study}

In this section, we present the dynamics of the new discrete model. The difference between the actual data and the numerical solution of the model is given, which demonstrates the effectiveness of the model. Then the prediction of the future behavior of the model is shown. By our model, it is reliable to make an estimation of the considered macroeconomic variable in the next few years.

\subsection{Data description}

In discrete model (8), the GDP $(x)$, exchange rate $(y)$, money supply $(z)$ and exports/imports $(u)$ are governed by a nonlinear dynamical system involving power laws. We preprocess the unit and scale of all data such that they are reasonable in a coupled nonlinear 
Table 1 Parameter sets in new discrete model (8)

\begin{tabular}{|c|c|c|c|c|c|c|c|}
\hline$\underline{P_{x}}$ & Value & $P_{y}$ & Value & $P_{z}$ & Value & $\boldsymbol{P}_{\boldsymbol{u}}$ & Value \\
\hline$\alpha_{1}$ & 8.22 & $\alpha_{2}$ & $-1.21 \mathrm{E}-02$ & $\alpha_{3}$ & 33.27 & $\alpha_{4}$ & $6.85 \mathrm{E}-05$ \\
\hline$h_{1}$ & 0.15 & $h_{2}$ & 5.63 & $h_{3}$ & 0.12 & $h_{4}$ & 39.47 \\
\hline$a_{11}$ & 28638.61 & $a_{21}$ & 24.82 & $a_{31}$ & 1969186.23 & $a_{41}$ & -0.97 \\
\hline$a_{12}$ & -58927.57 & $a_{22}$ & -12.20 & $a_{32}$ & 11605222.53 & $a_{42}$ & -0.62 \\
\hline$a_{13}$ & 5641.35 & $a_{23}$ & 0.29 & $a_{33}$ & -62813.00 & $a_{43}$ & $5.80 \mathrm{E}-03$ \\
\hline$a_{14}$ & -1113498.44 & $a_{24}$ & -9.75 & $a_{34}$ & 164429009.80 & $a_{44}$ & -5.08 \\
\hline$c_{1}$ & 1141119.70 & $c_{2}$ & -24.61 & $c_{3}$ & -212825070.60 & $c_{4}$ & 66.17 \\
\hline$\beta_{1}$ & 8.28 & $\beta_{2}$ & 1.12 & $\beta_{3}$ & 15.00 & $\beta_{4}$ & -0.31 \\
\hline
\end{tabular}

Figure 1 The actual data series of macroeconomic variables.

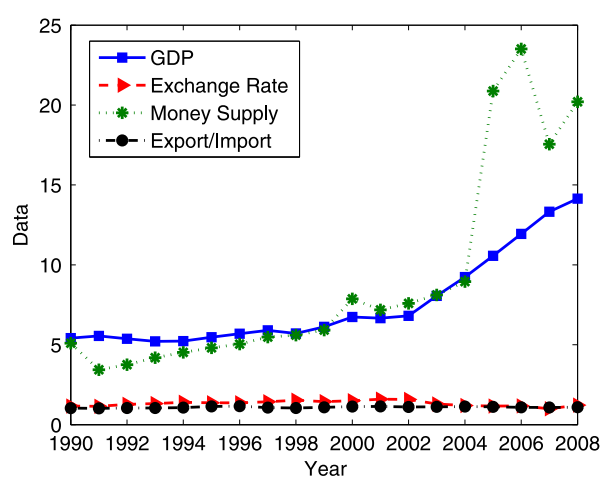

system. The annual data starts from year 1990 and continues to 2008. The unit of variables $x$ and $z$ is thousand billion US dollars and thousand billion Canadian dollars, respectively. The original data of these four macroeconomic variables are downloaded from http://data.worldbank.org.cn/indicator.

\subsection{Dynamics of the new discrete macroeconomic model}

Based on the actual data of Canada from 1990 to 2008, and taking the initial condition as

$$
\left(x_{0}, y_{0}, z_{0}, u_{0}\right)=(5.4115,1.16,5.1,1.0356) \text {, }
$$

the above extreme problem (10) is solved in Matlab, and the estimated parameters are given in Table 1. Substituting the coefficients and parameters into (8), the dynamics of our discrete fractal model can be easily calculated.

The actual data of the considered macroeconomic variables of Canada from 1990 to 2008 are shown in Figure 1. From this figure, we can observe that in the recent ten years, Canada has gained considerable development which is reflected by the growing GDP and money supply. The exchange rate and the ratio of exports and imports maintain an average level with the previous years.

Remark 1 We take the data from the period 1990 to 2008 mainly because those data are available in the Internet. The data of 2009 and 2010 will be used to make comparison with the empirical data given by the new model, which we can observe demonstrates the effectiveness and robustness of the new discrete fractal macroeconomic model.

To illustrate the effectiveness of model (8), the empirical simulation of macroeconomic variables is given in Figures 2-5. In Figure 2, we may see that the simulation data fit the ac- 
Figure 2 Comparison between actual and simulation data of GDP.

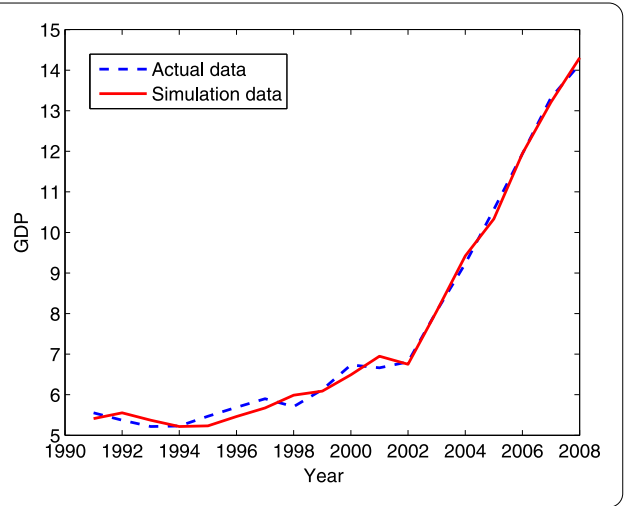

Table 2 Statistical test values on estimation of variable $x$

\begin{tabular}{ll}
\hline Name & Value \\
\hline Mean squared error & 0.1804 \\
Sum of squared error & 0.5859 \\
Correlation coefficient $(R)$ & 0.9980 \\
$R^{2}$ & 0.9961 \\
Determination coefficient & 0.9960 \\
Chi-square & 0.0447 \\
\hline
\end{tabular}

tual data very well, especially since 2002 . Even though the simulation curve parts from the actual curve little, in the view of least square it is still an optimal estimation. The statistical parameters in the method of least squares are shown in Table 2. We read that the mean squared error is small comparing to the average value of actual data of $x$. The correction coefficient indicates that the fitting data of GDP describe the actual data in an excellent way. The chi-squared result is 0.0447 , which is less than $\mathfrak{X}(1,0.05)=3.84$. It says that our model is reasonable under the confidence level of $95 \%$. The F-statistic result is 4037.0454 , which is greater than the critical value $\mathfrak{F}_{0.05}(1,1)=161.4$. It further demonstrates that the fractal model indeed describes the actual data. In practice, we usually consider the chisquared result as priority. If it is less than the critical value of some particular confidence level, then the result shows how accurate the constructed model is.

In Figure 3, it is illustrated that the empirical simulation satisfies the actual data very well. From the curves, there are some differences between 1994 and 2002, but this does not go against the trend of evolution. In 1993 and 1994, our simulation even gives the results pretty close to the actual data. The statistical parameters in the method of least squares are shown in Table 3. We observe that the mean squared error is 0.0883 , which leads the relative error to be in between $0.0883 / 1.6$ and $0.0883 / 0.9(5.52 \%-9.81 \%)$. The correction coefficient is 0.8360 , which implies that the fitting data of exchange rate is also effective. The chi-squared result is 0.0525 , which is less than $\mathfrak{X}(1,0.05)=3.84$. It shows that the fitting curve of variable $y$ in our model is reasonable under the confidence level of $95 \%$.

Figure 4 shows the fitting data and actual data of money supply, where we see that our model gives a very nice numerical simulation. From year 2002, these two curves are coincident with each other and there is no evident difference between them. The data of 1991 and 2002 have some deviation; however, the relative errors are acceptable comparing to the final values that the money supply arrives at. The statistical parameters in the method of least squares are shown in Table 4. We read that the mean squared error is 0.8443 , 

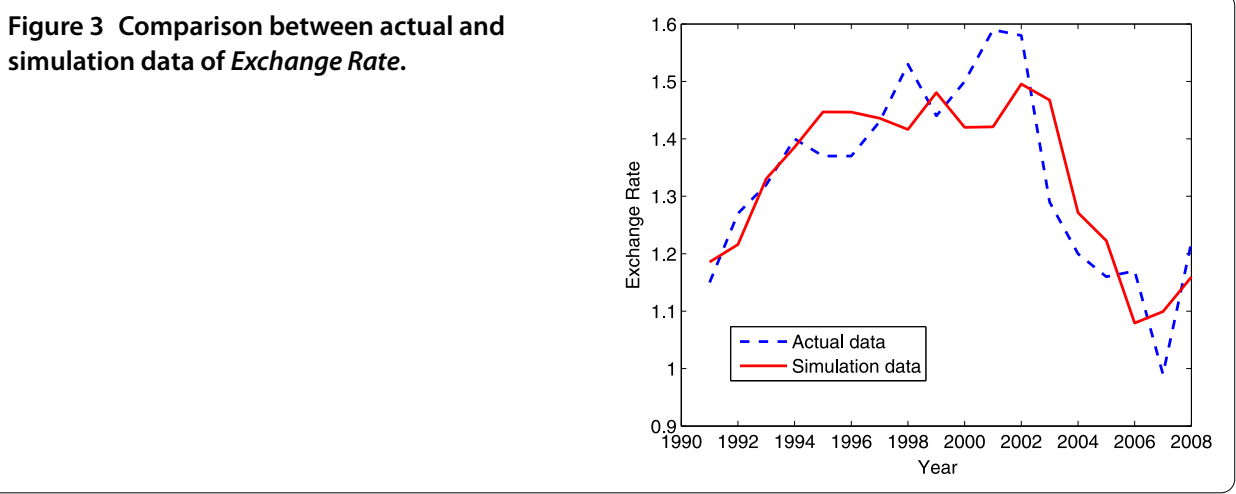

Table 3 Statistical test values on estimation of variable $y$

\begin{tabular}{ll}
\hline Name & Value \\
\hline Mean squared error & 0.0883 \\
Sum of squared error & 0.1403 \\
Correlation coefficient $(R)$ & 0.8360 \\
$R^{2}$ & 0.6989 \\
Determination coefficient & 0.6989 \\
Chi-square & 0.0525 \\
\hline
\end{tabular}

Figure 4 Comparison between actual and simulation data of Money Supply.

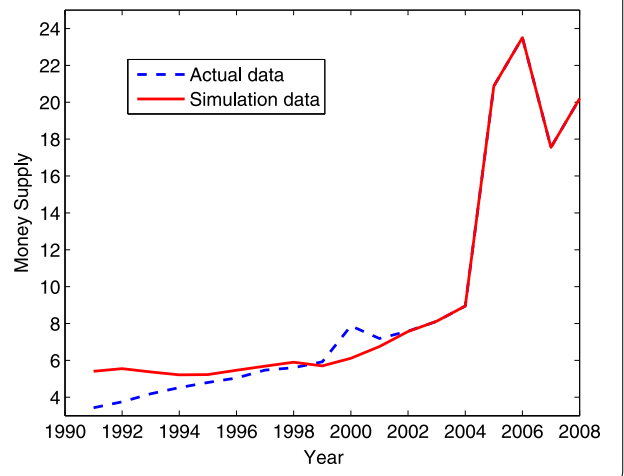

Year

Table 4 Statistical test values on estimation of variable $z$

\begin{tabular}{lr}
\hline Name & \multicolumn{1}{c}{ Value } \\
\hline Mean squared error & 0.8443 \\
Sum of squared error & 12.8308 \\
Correlation coefficient $(R)$ & 0.9924 \\
$R^{2}$ & 0.9848 \\
Determination coefficient & 0.9823 \\
Chi-square & 1.2705 \\
\hline
\end{tabular}

which shows that the fitting of our model is very nice. The correction coefficient is higher than that of the GDP and exchange rate, which indicates that the numerical simulation of money supply is better than that of GDP and exchange rate. The chi-squared result is 1.2705 , which is less than $\mathfrak{X}(1,0.05)=3.84$. It says that the fitting curve of variable $z$ in our model is reliable under the confidence level of $95 \%$.

As before, similar observations can be made in Figure 5. We can see that the empirical simulation of exports/imports fits the actual data very well, in particular see years after 
Figure 5 Comparison between actual and simulation data of Exports/Imports.

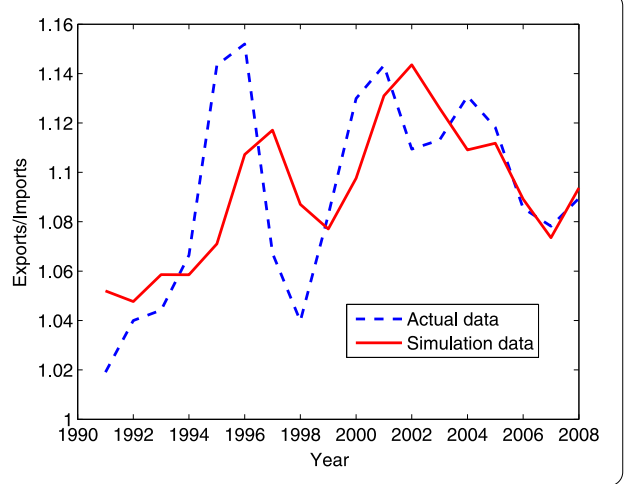

Table 5 Statistical test values on estimation of variable $u$

\begin{tabular}{ll}
\hline Name & Value \\
\hline Mean squared error & 0.0265 \\
Sum of squared error & 0.0126 \\
Correlation coefficient $(R)$ & 0.7380 \\
$R^{2}$ & 0.5476 \\
Determination coefficient & 0.9823 \\
Chi-square & 0.0057 \\
\hline
\end{tabular}

Table 6 Prediction of macroeconomic variables in 2009 to 2010

\begin{tabular}{lcllc}
\hline Variable & GDP & Exchange rate & Money supply & Exports/Imports \\
\hline Prediction in 2009 & 14.602 & 1.21 & 22.424 & 1.0921 \\
Actual data in 2009 & 12.525 & - & - & $3166 / 3299$ \\
Error & 2.077 & - & - & 0.1324 \\
Relative error & $14.22 \%$ & - & - & 12.12 \\
Prediction in 2010 & 14.835 & 1.08 & 21.073 & 1.1081 \\
Actual data in 2010 & 14.797 & - & - & $3879 / 4025$ \\
Error & 0.037 & - & - & 0.1443 \\
Relative error & $2.49 \%$ & - & - & $13.02 \%$ \\
\hline
\end{tabular}

2005. The statistical parameters in the method of least squares are shown in Table 5. We notice that the mean squared error is 0.0265 , and the sum of squared error is 0.0126 , which demonstrates that the numerical simulation of our model is effective. The correction coefficient is 0.7380 , which also indicates that the fitting curve coincides with the actual data well. The chi-squared result is 0.0057 , which is less than $\mathfrak{X}(1,0.05)=3.84$. It says that the fitting curve of variable $u$ in our model is reasonable under the confidence level of $95 \%$.

\subsection{Prediction of the future evolution}

To verify the robustness of model (8), we predict the values of the GDP, exchange rate, money supply, and exports/imports in the next two years, i.e., in 2009 and 2010. The errors between the numerical simulations and the actual data are given in Table 6 , where we can observe that our discrete model (8) gives an ideal prediction. Due to the fact that the actual data of exchange rate and money supply of 2009 and 2010 are not available, we only give the evaluation and comparison of GDP and Exports/Imports in those two years. We may see that the relative error is around $10 \%$ to $15 \%$, and in some cases our model predicts the future evolution with relative error less than $2.50 \%$, which is remarkable. 


\section{Conclusion}

In this paper, we proposed a novel nonlinear discrete macroeconomic model based on the generalized fractal derivatives. The advantage of employing fractal derivative consists of two aspects. One is that the generalized fractal derivative is suitable for depicting the power law in macroeconomic variables. The other is that the difference form of generalized fractal derivative has finite term, which is different from the fractional derivative in Riemann-Liouville, Caputo, and Riesz senses, where their difference expressions involve infinitely many terms as the step size goes to infinity. In our new model, the step size in discretization and the order in fractal derivative are regarded as parameters in the obtained discrete equation. All the parameters are estimated by the least-squares method. Based on the macroeconomic data, we calculate the optimal parameters in fractal derivatives and step size. The sum squared residuals and the mean squared errors of estimation in simulation are computed, which demonstrate that the nonlinear discrete model is effective in modeling the macroeconomic variables of Canada. It would be expected that the nonlinear discrete model proposed in this paper is better than the linear model proposed in previous references.

\section{Additional remarks}

Now we would like to make several additional remarks based on our research experience of this work:

- As a similar topic, fractional calculus has been applied in many scientific areas and engineering fields. In economic and financial realms, people found that the fractional derivative modeling can describe the memory property in many different financial series. However, as we stated, the power-law phenomenon is also one of the obvious characteristics that lots of macroeconomic variables usually exhibit. The fractal modeling, as a strong mathematical tool in studying the power-law feature, has not been applied widely. In our research, we show that the fractal derivative modeling can be used to investigate the coupled relation of different macroeconomic variables.

- The modeling method discussed in this paper is nonstandard or unique. We may obtain other discrete models which are different from equation (9) by using other approximation scheme in the generalized fractal derivative.

- Although we only consider the macroeconomic variables, i.e., GDP, exchange rate, money supply and exports/imports of Canada, many other inner-connected macroeconomic variables can be considered using the same method. Our modeling methodology can also be applied to other countries to investigate their evolution of different macroeconomic variables. 


\section{References}

1. Kilbas, AA, Srivastava, HM, Trujillo, JJ: Theory and Applications of Fractional Differential Equations. Elsevier, Amsterdam (2006)

2. Podlubny, I: Fractional Differential Equations. Academic Press, San Diego (1999)

3. Diethelm, K: The Analysis of Fractional Differential Equations. Springer, Berlin (2010)

4. $\mathrm{Ma}, \mathrm{S}, \mathrm{Xu}, \mathrm{Y}, \mathrm{Yue}, \mathrm{W}$ : Numerical solutions of a variable-order fractional financial system. J. Appl. Math. 2012, Article ID 417942 (2012). doi:10.1155/2012/417942

5. Ma, S, Xu, Y, Yue, W: Existence and uniqueness of solution for a class of nonlinear fractional differential equations. Adv. Differ. Equ. 2012, Article ID 133 (2012)

6. $\mathrm{Xu}, \mathrm{YF}, \mathrm{He}, \mathrm{ZM}$ : Synchronization of variable-order fractional financial system via active control method. Cent. Eur. J. Phys. 11(6), 824-835 (2013)

7. Xu, YF, Agrawal, OP: Models and numerical schemes for generalized van der Pol equations. Commun. Nonlinear Sci. Numer. Simul. 18, 3575-3589 (2013)

8. $\mathrm{Hu}, \mathrm{ZH}, \mathrm{Chen}, \mathrm{W}$ : Modeling of macroeconomics by a novel discrete nonlinear fractional dynamical system. Discrete Dyn. Nat. Soc. 2013, Article ID 275134 (2013)

9. Yue, YD, He, L, Liu, GC: Modeling and application of a new nonlinear fractional financial model. J. Appl. Math. 2013, Article ID 325050 (2013). doi:10.1155/2013/325050

10. He, JH: A tutorial review on fractal spacetime and fractional calculus. Int. J. Theor. Phys. 53(11), 3698-3718 (2014)

11. Yang, XJ, Baleanu, D: Fractal heat conduction problem solved by local fractional variation iteration method. Therm. Sci. 17(2), 625-628 (2013)

12. Yang, XJ, Srivastava, HM, He, JH, Baleanu, D: Cantor-type cylindrical-coordinate method for differential equations with local fractional derivatives. Phys. Lett. A 377(28-30), 1696-1700 (2013)

13. Baleanu, D, Machado, JAT, Cattani, C, Baleanu, MC, Yang, XJ: Local fractional variational iteration and decomposition methods for wave equation on Cantor sets within local fractional operators. Abstr. Appl. Anal. 2014, Article ID 535048 (2014). doi:10.1155/2014/535048

14. Srivastava, HM, Golmankhaneh, AK, Baleanu, D, Yang, XJ: Local fractional Sumudu transform with application to IVPS on Cantor sets. Abstr. Appl. Anal. 2014, Article ID 620529 (2014)

15. Yang, XJ, Baleanu, D, Zhong, WP: Approximate solutions for diffusion equations on Cantor space-time. Proc. Rom Acad., Ser. A: Math. Phys. Tech. Sci. Inf. Sci. 14(2), 127-133 (2013)

16. Baleanu, D, Srivastava, HM, Yang, XJ: Local fractional variational iteration algorithms for the parabolic Fokker-Planck equation defined on Cantor sets. Progr. Fract. Differ. Appl. 1(1), 1-11 (2015)

17. Farmer, JD, Geanakoplos, J: Power laws in finance and their implications for economic theory. Mimeo, Santa Fe Institute, Santa Fe (2004)

18. Gabaix, X, Gopikrishnan, P, Plerou, V, Stanley, HE: A theory of power-law distributions in financial market fluctuations Nature 423(6937), 267-270 (2003)

19. Gabaix, X, Gopikrishnan, P, Plerou, V, Stanley, HE: Institutional investors and stock market volatility. Q. J. Econ. 121(2), 461-504 (2006)

20. Rak, R, Drożdż, S, Kwapień, J, Oświęcimka, P: Stock returns versus trading volume: is the correspondence more general? Acta Phys. Pol. B 44(10), 2035-2050 (2013)

21. Aharon, B, Solomon, S: Power laws in cities population, financial markets and Internet sites (scaling in systems with a variable number of components. Physica A 287(1), 279-288 (2000)

22. Chian, ACL, Borotto, FA, Rempel, EL, Rogers, C: Attractor merging crisis in chaotic business cycles. Chaos Solitons Fractals 24(3), 869-875 (2005)

23. $\mathrm{Ma}, \mathrm{JH}$, Chen, YS: Study for the bifurcation topological structure and the global complicated character of a kind of nonlinear finance system. Appl. Math. Mech. 22(11), 1240-1251 (2001)

24. $\mathrm{Ma}, \mathrm{JH}$, Chen, YS: Study for the bifurcation topological structure and the global complicated character of a kind of nonlinear finance system. II. Appl. Math. Mech. 22(12), 1375-1382 (2001)

25. Chen, WC: Nonlinear dynamics and chaos in a fractional order financial system. Chaos Solitons Fractals 36(5), 1305-1314 (2008)

26. Dadras, S, Momeni, HR: Control of a fractional-order economical system via sliding mode. Physica A 389(12), 2434-2442 (2010)

27. Wang, Z, Huang, $X$, Shi, G: Analysis of nonlinear dynamics and chaos in a fractional order financial system with time delay. Comput. Math. Appl. 62(3), 1531-1539 (2011)

28. Skovranek, T, Podlubny, I: Modeling of the national economics in state-space: a fractional calculus approach. Econ. Model. 29(4), 1322-1327 (2012)

29. Agarwal, RP: Difference Equations and Inequalities: Theory, Methods and Applications. CRC Press, Boca Raton (2000)

30. Wu, G-C, Baleanu, D: Discrete fractional logistic map and its chaos. Nonlinear Dyn. 72(1-2), 283-287 (2014)

31. Wu, G-C, Baleanu, D: Chaos synchronization of the discrete fractional logistic map. Signal Process. 102, 96-99 (2014)

32. Chen, W: Time-space fabric underlying anomalous diffusion. Chaos Solitons Fractals 28(4), 923-929 (2006)

33. Chen, W, Sun, HG, Zhang, X, Korosak, D: Anomalous diffusion modeling by fractal and fractional derivatives. Comput. Math. Appl. 59(5), 1754-1758 (2010)

34. Tatom, FB: The relationship between fractional calculus and fractals. Fractals 3(1), 217-229 (1995)

35. Kanno, R: Representation of random walk in fractal space-time. Physica A 248(1), 165-175 (1998) 